\title{
CONTROL OF CONSTRAINED NONLINEAR UNCERTAIN DISCRETE-TIME SYSTEMS VIA ROBUST CONTROLLABLE SETS: A MODAL INTERVAL ANALYSIS APPROACH
}

\author{
Jian Wan ${ }^{1}$, Josep Vehí ${ }^{1}$, Ningsu LuO $^{1}$ and Pau Herrero ${ }^{1}$
}

\begin{abstract}
A general framework for computing robust controllable sets of constrained nonlinear uncertain discrete-time systems as well as controlling such complex systems based on the computed robust controllable sets is introduced in this paper. The addressed one-step control approach turns out to be a robust model predictive control scheme with feasible unit control horizon and contractive constraint. The solver of 1-dimensional quantified set inversion in modal interval analysis is extended to 2-dimensional cases for computing robust controllable sets off-line with a clear semantic interpretation, where both universal and existential quantifiers are concerned simultaneously. An interval-based solver of constrained minimax optimization is also proposed to compute one-step control inputs online in a reliable way, which guarantee to drive the system state contractively along the computed robust controllable sets to a selected terminal robust control invariant set.
\end{abstract}

Mathematics Subject Classification. 65G40, 93B05, 93B51.

Received December 12, 2006. Revised June 11, 2007.

Published online March 28, 2008.

\section{INTRODUCTION}

A fundamental control problem is to determine the subset of the state space that can be steered via an admissible control sequence to a given terminal set while guaranteeing that the state constraint be satisfied for all uncertain cases. Such a subset is referred to as a robust controllable set and it is a more general interpretation of the classical controllability problem of unconstrained deterministic linear systems [10]. The given terminal set is usually selected to be a robust control invariant set for the system, i.e., there always exists an admissible control input according to the related local stabilizing feedback control law that guarantees to keep the system state inside the robust control invariant set for all uncertain cases [14]. The properties of robust control invariant sets are involved in various control problems such as constrained control, robustness synthesis and constrained optimization [1].

The analytical determination of controllable sets as well as the controllability of constrained discrete-time systems is generally difficult. In [18], zero-controllability of linear discrete-time systems under control actions bounded in magnitude and norm was discussed analytically. The controllability of constrained nonlinear

\footnotetext{
Keywords and phrases. Nonlinearity, uncertainty, constraints, robust controllable set, quantified set inversion, minimax optimization, interval analysis, modal intervals.

1 Institut d'Informàtica i Aplicacions, Universitat de Girona, Campus Montilivi, 17071 Girona, Spain. jwan@eia.udg.edu; vehi@eia.udg.edu; ningsu@eia.udg.edu; pherrero@eia.udg.edu
} 
discrete-time systems was also discussed analytically in [11], where primary results on local constrained controllability problems were obtained by using some mapping theorems taken from nonlinear functional analysis and linear approximation methods. These analytical approaches for the controllability of constrained discrete-time systems were narrowed with specific assumptions because systematic and explicit controllability analysis for general constrained discrete-time systems is extremely hard.

A numerical approach for determining the controllability of constrained discrete-time systems is to compute controllable sets geometrically and any initial state within the computed maximal controllable set is controllable to the selected terminal set in finite steps [10]. In [16], robust controllable sets were computed geometrically via a set of set differences and projections for constrained discrete-time systems with additive uncertainty. Such an approach is quite effective for discrete-time linear or piecewise-affine systems with polytopes as their terminal sets, where the set computations can be performed efficiently through polyhedral algebra, linear programming and computational geometry software. However, the computation of robust controllable sets for general constrained nonlinear discrete-time systems is not straightforward and efficient. In [2], a branch-and-bound algorithm based on interval arithmetic was introduced to compute the inner approximations of control invariant sets for constrained nonlinear systems. This algorithm was extended to compute robust controllable sets for constrained nonlinear systems with additive uncertainty in [13], where the computed robust controllable sets were utilized as a contractive sequence of sets to formulate a contractive model predictive control scheme for simplifying overwhelming minimax optimizations underlying robust model predictive control. However, bisections and selections of the state space and the admissible control space were mixed in their approaches, where extra treatments of the subboxes of the overall domain were needed after each bisection and selection of the state space.

This paper deals with the computation of robust controllable sets for constrained nonlinear discrete-time systems with parametric or additive uncertainty, where the interval vector for the state space, the interval vector for the admissible control space and the interval vector for the uncertainty are all considered as modal interval vectors with corresponding modalities [5,7]. Thus bisections and selections of the state space and the admissible control space are to be separated by two nested loops during the computation of robust controllable sets via the generalized solver of 2-dimensional quantified set inversion, where a clear semantic interpretation for bisections and selections of the state space exists and extra treatments of the subboxes of the overall domain are avoided after each bisection and selection of the state space. The computed robust controllable sets are also utilized as a contractive sequence of sets to formulate a one-step control scheme for simplifying traditional multi-step minimax optimizations underlying robust model predictive control. The simplified one-step minimax optimizations are fulfilled by a proposed solver of constrained minimax optimization in a reliable way.

The paper is organized as follows: the problem considered is stated in Section 2; modal interval analysis and its solvers are introduced in a comparative way relative to classical interval analysis in Section 3 ; the robust controllable set approximation algorithm based on the generalized solver of 2-dimensional quantified set inversion, the one-step control algorithm based on the computed robust controllable sets and the intervalbased solver of constrained minimax optimization, and feasibility and stability analysis of the addressed control approach are described in Section 4; an illustrative example is provided in Section 5; and finally, some conclusions are drawn in Section 6.

\section{Problem statement}

The system to be considered is described by the following constrained nonlinear discrete-time state-space model with parametric or additive uncertainty:

$$
\mathbf{x}(k+1)=\mathbf{f}(\mathbf{x}(k), \mathbf{w}(k), \mathbf{u}(k)), k=0, \ldots,
$$

where $\mathbf{x}(k) \in \mathbf{X} \subset \mathbb{R}^{n}$ is a vector of $n$ state variables and $\mathbf{X}$ is a compact set containing the origin; w $(k) \in \mathbf{W} \subset$ $\mathbb{R}^{l}$ is a vector of $l$ uncertain parameters or additive disturbances; $\mathbf{u}(k) \in \mathbf{U} \subset \mathbb{R}^{m}$ is a vector of $m$ control inputs and $\mathbf{U}$ is a compact set containing the origin. The domains of $\mathbf{X}, \mathbf{W}$ and $\mathbf{U}$ are assumed to be described by boxes, 
i.e., every component of the vectors is an interval. Such a model represents a general class of practical systems with constrained state and control as well as uncertain parameters or additive disturbances. The control target is to drive the system from the initial state $\mathbf{x}(0)$ to a sufficiently small region around the origin asymptotically. The dual-mode approach of model predictive control is adopted here: at first, the one-step control derived from model predictive control with a contractive sequence of robust controllable sets drives the system state into a selected terminal robust control invariant set $\mathbb{T}$; and then the related local stabilizing feedback control law is applied instead to drive the system state to a sufficiently small region around the origin asymptotically. The selected terminal robust control invariant set as well as the related local stabilizing feedback control law can be designed in advance using the methods proposed in $[3,4]$.

The one-step robust controllable set $\tilde{\mathcal{K}}(\mathbf{X}, \mathbb{T})$ is the set of states in $\mathbf{X}$ within which an admissible control input $\mathbf{u}(k) \in \mathbf{U}$ exists that guarantees to drive the system state to the selected terminal set $\mathbb{T}$ in one step for every allowable uncertainty $\mathbf{w}(k) \in \mathbf{W}$, i.e.,

$$
\tilde{\mathcal{K}}(\mathbf{X}, \mathbb{T})=\{\mathbf{x}(k) \in \mathbf{X} \mid \forall \mathbf{w}(k) \in \mathbf{W}, \exists \mathbf{u}(k) \in \mathbf{U}: \mathbf{x}(k+1) \in \mathbb{T}\}
$$

It can be seen that both universal and existential quantifiers are concerned simultaneously for various kinds of interval vectors in the definition of one-step robust controllable sets, which motivates the application of modal intervals to deal with the computation of robust controllable sets in an intrinsic way. The selected terminal set $\mathbb{T}$ is a robust control invariant set if and only if $\mathbb{T} \subseteq \tilde{\mathcal{K}}(\mathbf{X}, \mathbb{T})[10,14]$. If the terminal set $\mathbb{T}$ is selected to be a robust control invariant set, then the one-step robust controllable set $\tilde{\mathcal{K}}(\mathbf{X}, \mathbb{T})$ can also be referred to as the one-step robust stabilisable set $[10,16]$. The $i$ th-step robust controllable set $\tilde{\mathcal{K}}_{i}(\mathbf{X}, \mathbb{T})$ can be obtained further by computing one-step robust controllable sets recursively, i.e.,

$$
\tilde{\mathcal{K}}_{i}(\mathbf{X}, \mathbb{T})=\tilde{\mathcal{K}}\left(\mathbf{X}, \tilde{\mathcal{K}}_{i-1}(\mathbf{X}, \mathbb{T})\right)
$$

where $\tilde{\mathcal{K}}_{0}(\mathbf{X}, \mathbb{T})=\mathbb{T}$ and the maximal robust controllable set $\tilde{\mathcal{K}}_{\infty}(\mathbf{X}, \mathbb{T})$ within the constrained state space $\mathbf{X}$ is reached when $\tilde{\mathcal{K}}_{N+1}(\mathbf{X}, \mathbb{T})=\tilde{\mathcal{K}}_{N}(\mathbf{X}, \mathbb{T})$ for certain $N$.

Once the maximal robust controllable set $\tilde{\mathcal{K}}_{N}(\mathbf{X}, \mathbb{T})$ is obtained, the controllability of the constrained nonlinear uncertain discrete-time system (2.1) is obvious: the system is robustly controllable to the selected terminal set $\mathbb{T}$ in finite steps if the initial state is within the maximal robust controllable set $\tilde{\mathcal{K}}_{N}(\mathbf{X}, \mathbb{T})$. The control inputs can be obtained through the strategy of robust model predictive control with feasible unit control horizon and contractive constraint when the system state is outside the selected terminal set $\mathbb{T}$, i.e., the one-step control inputs are to be obtained by solving the following constrained minimax optimization iteratively:

$$
\mathbf{u}^{\text {Optimal }}(k)=\arg \min _{\mathbf{u}(k) \in \mathbf{U}} \max _{\mathbf{w}(k) \in \mathbf{W}}\left[\mathbf{x}^{T}(k+1) Q \mathbf{x}(k+1)+\mathbf{u}^{T}(k) R \mathbf{u}(k)\right]
$$

subject to

$$
\mathbf{x}(k+1) \in \tilde{\mathcal{K}}_{i-1}(\mathbf{X}, \mathbb{T})
$$

where the initial sate $\mathbf{x}(k) \in \tilde{\mathcal{K}}_{i}(\mathbf{X}, \mathbb{T})$, but $\mathbf{x}(k)$ does not belong to $\tilde{\mathcal{K}}_{i-1}(\mathbf{X}, \mathbb{T}) ; Q$ and $R$ are weighted positive definite matrices for the cost function; and $\mathbf{u}^{\text {Optimal }}(k)$ is the obtained optimal one-step control input.

\section{MODAl INTERVAL ANALYSiS}

The initial idea of classical interval analysis is to enclose real numbers in intervals and real vectors in boxes as a method of considering the imprecision of representing real numbers by finite numbers of digits in numerical computers. Classical interval analysis has become a fundamental numerical tool for representing uncertainties or errors, proving properties of sets, solving sets of nonlinear equations or inequalities and optimizing in a global way $[6,8]$. Classical interval arithmetic is based on four elementary arithmetic operations $(+,-, \times, \div)$ 
on intervals rather than on real numbers. An essential property of the natural inclusion function $F(\mathbf{X})$ of a continuous function $f: \mathbb{R}^{n} \rightarrow \mathbb{R}$ over a box $\mathbf{X} \in \mathbb{I}\left(\mathbb{R}^{n}\right)$ is:

$$
f(\mathbf{X}) \subseteq F(\mathbf{X})
$$

where $\mathbb{I}\left(\mathbb{R}^{n}\right)$ is denoted to the set of all $n$-dimensional classical interval vectors; $f(\mathbf{X})=\left[\min _{\mathbf{x} \in \mathbf{X}} f(\mathbf{x}), \max _{\mathbf{x} \in \mathbf{X}}\right.$ $f(\mathbf{x})]$ is the classical interval function; and the natural inclusion function $F(\mathbf{X})$ of $f(\mathbf{x})$ is obtained by replacing each occurrence of every variable with the corresponding interval variable, by executing all elementary operations according to interval arithmetic, and by computing ranges of the standard functions [15]. The semantic statement for (3.1) is:

$$
\forall x_{1} \in\left[a_{1}, b_{1}\right] \ldots \forall x_{n} \in\left[a_{n}, b_{n}\right] \exists z \in F(\mathbf{X}) z=f\left(x_{1}, \ldots, x_{n}\right)
$$

The classical interval function $f(\mathbf{X})$ can seldom be computed exactly via interval arithmetic. Its natural inclusion function $F(\mathbf{X})$, which can be computed directly via interval arithmetic, is utilized instead in various classical interval solvers such as set inversion and global optimization, where the bisection of interval vectors and the selection of appropriate interval vectors are performed according to the lower and upper bounds of $F$. Taking the problem of set inversion as an example, for a possibly nonlinear function $\mathbf{f}(\mathbf{x}): \mathbb{R}^{n} \rightarrow \mathbb{R}^{m}$ with the initial domain $\mathbf{X} \subset \mathbb{R}^{n}$ and the known range $\mathbb{Y} \subset \mathbb{R}^{m}$, set inversion is the characterization [8]:

$$
\mathbb{X}=\{\mathbf{x} \in \mathbf{X} \mid \mathbf{f}(\mathbf{x}) \in \mathbb{Y}\}=\mathbf{f}^{-1}(\mathbb{Y}) \cap \mathbf{X}
$$

The solver of set inversion via interval analysis is an algorithm of set computation aiming to find the feasible solution $\mathbb{X} \subseteq \mathbf{X}$ satisfying $\mathbf{f}(\mathbb{X}) \subseteq \mathbb{Y}$. It is fulfilled in a numerical way by bisecting the initial domain $\mathbf{X}$ of $\mathbf{f}(\mathbf{x})$ into subboxes, computing the inclusion function $\mathbf{F}$ of $\mathbf{f}(\mathbf{x})$ for each subbox via interval arithmetic, and finally selecting all feasible subboxes through comparing the resulting image of the inclusion function $\mathbf{F}$ for each subbox with the known range $\mathbb{Y}[8]$.

The computation of control invariant sets in [2] can be regarded as a direct application of the solver of set inversion via interval analysis, where the selected terminal set was the known range $\mathbb{Y}$ in $(3.3)$ and the initial domain $\mathbf{L}=(\mathbf{X}, \mathbf{U})$ was the combination of the state space $\mathbf{X}$ and the admissible control space $\mathbf{U}$. However, these two interval vectors have different modalities: for a subbox of the state space, it belongs to a controllable set if and only if for all states within the subbox there always exists an admissible control input that can drive them to the selected terminal set; for a subbox of the admissible control space, it is a feasible control domain if and only if there exists one value within the subbox which can drive the concerned subbox of the state space to the selected terminal set. The bisection and the selection of the state space and the admissible control space were mixed in [2], where extra treatments of the subboxes of the overall domain were needed after each bisection and selection of the state space. The extra treatments of the subboxes of the overall domain were time-consuming because the members of the overall domain were numerous and the action of bisection and selection was frequent. Furthermore, there was no semantic interpretation for discarding the subbox of the state space: if the action for $\mathbf{L}_{k}=\left(\mathbf{X}_{k}, \mathbf{U}_{k}\right)$ was to discard, this did not mean that $\mathbf{X}_{k}$ could not be a part of the one-step controllable set because $\mathbf{X}_{k}$ was likely to be controllable to the selected terminal set for other parts of the admissible control space $\mathbf{U}$.

Modal interval analysis is an extension of classical interval analysis obtained by distinguishing existential and universal modalities of intervals encountered in practical problems such as the computation of controllable sets. The following subsections give a comprehensive and tutorial introduction of modal interval analysis and the solvers of quantified set inversion and constrained minimax optimization. Every concept of modal interval analysis is derived by extending a counterpart concept of classical interval analysis and modal interval analysis is treated as an extension of classical interval analysis in modality, inclusion, semantics and arithmetic. 


\subsection{The initiative of modal intervals - modal extension}

Intervals encountered in practical problems have two modalities: (1) there exists a value in $[a, b]$, (with $a \leq b)$ that possesses a property or some given properties; (2) all values in $[a, b]$, (with $a \leq b$ ) possess a property or some given properties. Classical interval analysis cannot distinguish these two types of intervals and denotes them as $[a, b]$, (with $a \leq b)$ uniformly. Modal interval analysis does distinguish these two types of intervals and denotes them differently: $[a, b]^{*}$, (with $a \leq b$ ) for those proper intervals that only require the existence of a value in the domain of $a \leq x \leq b$ to possess a property or some given properties; and $[b, a]^{*}$, (with $a \leq b$ ) for those improper intervals that require all values in the domain of $a \leq x \leq b$ to possess a property or some given properties, where $[a, b]^{*}$ and $[b, a]^{*}$ are modal intervals ${ }^{1}$. A modal interval $[a, b]^{*}$ is therefore a pair of a classical interval and a corresponding modality [5], i.e.,

$$
[a, b]^{*}:=\left(\operatorname{Prop}\left([a, b]^{*}\right), Q\left([a, b]^{*}\right)\right)=\left\{\begin{array}{l}
([a, b], \exists) \text { if } a \leq b \\
([b, a], \forall) \text { if } a \geq b
\end{array}\right.
$$

where $\operatorname{Prop}\left([a, b]^{*}\right)=[\min \{a, b\}, \max \{a, b\}]$ is the classical interval domain and $\mathrm{Q}\left([a, b]^{*}\right) \in\{\forall, \exists\}$ is the modality of $[a, b]^{*}$. The lower and upper bounds of $[a, b]^{*}$ are represented by $\operatorname{Lb}\left([a, b]^{*}\right)=a$ and $\mathrm{Ub}\left([a, b]^{*}\right)=b$, respectively. The operator Dual is to change the modality of a modal interval, i.e., $\operatorname{Dual}\left([a, b]^{*}\right)=[b, a]^{*}$. It is interesting to note that classical intervals are often treated implicitly as proper intervals with the modality of $\exists$ in classical interval analysis before the concept of modal intervals was proposed.

Accordingly, the modal interval vector $\mathbf{X}^{*} \in \mathbb{I}^{*}\left(\mathbb{R}^{n}\right)$ represents a vector whose components are all modal intervals, where $\mathbb{I}^{*}\left(\mathbb{R}^{n}\right)$ is denoted to the set of all $n$-dimensional modal interval vectors. The entries of $\mathbf{X}^{*}$ are usually partitioned into two sub-vectors according to the modality of every component:

$$
\mathbf{X}^{*}=\left(\mathbf{X}_{p}^{*}, \mathbf{X}_{i}^{*}\right)
$$

where $\mathbf{X}_{p}^{*}$ is composed of proper intervals with the modality $\exists$ and $\mathbf{X}_{i}^{*}$ is composed of improper intervals with the modality $\forall$. The basic operation of bisecting a classical interval vector can also be extended to modal interval vectors. Taking the modal interval vector $\mathbf{X}^{*}=\left[a_{1}, b_{1}\right]^{*} \times \ldots \times\left[a_{n}, b_{n}\right]^{*}$ as an example, its width is denoted to be:

$$
\operatorname{Width}\left(\mathbf{X}^{*}\right)=\max _{i=1, \ldots, n}\left|a_{i}-b_{i}\right|
$$

and the index $j$ is denoted to be:

$$
j=\min _{i=1, \ldots, n}\left\{i \mid\left(\left|a_{i}-b_{i}\right|\right)=\operatorname{Width}\left(\mathbf{X}^{*}\right)\right\}
$$

then the bisection Bisect $\left(\mathbf{X}^{*}\right)$ returns two sub-modal interval vectors $\mathrm{LX}^{*}$ and $\mathrm{RX}^{*}$ :

$$
\left\{\begin{array}{l}
\mathrm{LX}^{*}:=\left[a_{1}, b_{1}\right]^{*} \times \ldots \times\left[a_{j}, \frac{\left(a_{j}+b_{j}\right)}{2}\right]^{*} \times \ldots \times\left[a_{n}, b_{n}\right]^{*} \\
\mathrm{RX}^{*}:=\left[a_{1}, b_{1}\right]^{*} \times \ldots \times\left[\frac{\left(a_{j}+b_{j}\right)}{2}, b_{j}\right]^{*} \times \ldots \times\left[a_{n}, b_{n}\right]^{*} .
\end{array}\right.
$$

\subsection{The quantifier of modal intervals - inclusion extension}

The modality of a modal interval $[a, b]^{*}$ provides the quantifier for all properties possessed by the modal interval: for a proper interval, the quantifier is $\exists x \in \operatorname{Prop}\left([a, b]^{*}\right)$; and for an improper interval, the quantifier is $\forall x \in \operatorname{Prop}\left([a, b]^{*}\right)$. The inclusion between classical intervals $[a, b] \subseteq[c, d]$, (with $a \leq b, c \leq d$ ) can be interpreted as: if there exists a value $x \in[a, b]$ that possesses a property or some given properties, then there also exists

\footnotetext{
${ }^{1}$ In the seminal paper on modal intervals [5], $[a, b]$ represented a modal interval and $[a, b]^{\prime}$ represented a classical interval; in this paper, $[a, b]^{*}$ is a modal interval while the notation $[a, b]$ is kept to represent a classical interval.
} 
a value $y \in[c, d]$ that possesses the same property(properties). Accordingly, the inclusion between modal intervals or sets $[a, b]^{*} \subseteq[c, d]^{*}$ can be extended to be:

$$
[a, b]^{*} \subseteq[c, d]^{*} \Leftrightarrow \operatorname{Pred}\left([a, b]^{*}\right) \subseteq \operatorname{Pred}\left([c, d]^{*}\right)
$$

where $\operatorname{Pred}(\cdot)$ is denoted to the set of all properties possessed by a modal interval along with its quantifier. For example, the property smaller than 3.5 belongs to both $\operatorname{Pred}\left([3,2]^{*}\right)$ and $\operatorname{Pred}\left([1,4]^{*}\right)$ because $\forall x \in[2,3] x<3.5$ and $\exists x \in[1,4] x<3.5$. The extended modal interval inclusion (3.9) can be further simplified as [5]:

$$
[a, b]^{*} \subseteq[c, d]^{*} \Leftrightarrow(a \geq c, b \leq d)
$$

This can be demonstrated by considering the quantifiers of modal intervals and the definition of inclusion in (3.9). For instance, for the case of $a \geq b, c \leq d$, i.e., $\mathbf{Q}\left([a, b]^{*}\right)=\forall$ and $\mathbf{Q}\left([c, d]^{*}\right)=\exists$, $\operatorname{Pred}\left([a, b]^{*}\right) \subseteq \operatorname{Pred}\left([c, d]^{*}\right)$ means that there always exists a value in the domain of $c \leq y \leq d$ that possesses the same property(properties) possessed by all values in the domain of $b \leq x \leq a$, then $[b, a] \cap[c, d] \neq \emptyset$ must be satisfied and thus $a \geq c, b \leq d$ can be derived from $\operatorname{Pred}\left([a, b]^{*}\right) \subseteq \operatorname{Pred}\left([c, d]^{*}\right)$ as well as $[a, b]^{*} \subseteq[c, d]^{*}$, e.g., $[3,2]^{*} \subseteq[1,4]^{*}$. The inclusion conditions between modal interval vectors can also be deduced according to the definition of inclusion for each component of modal interval vectors.

Similar to classical intervals, the inner and outer representations of $[a, b]^{*} \in \mathbb{I}^{*}(\mathbb{R})$ by a fixed number of digits are defined to be:

$$
\left\{\begin{array}{l}
\operatorname{Inn}\left([a, b]^{*}\right)=[\operatorname{Right}(a), \operatorname{Left}(b)]^{*} \\
\operatorname{Out}\left([a, b]^{*}\right)=[\operatorname{Left}(a), \operatorname{Right}(b)]^{*},
\end{array}\right.
$$

where Left $(a)=\sup _{x} x \leq a, \operatorname{Right}(a)=\inf _{x} x \geq a$ and $x$ is represented by the fixed number of digits. It is obvious that

$$
\operatorname{Inn}\left([a, b]^{*}\right)=\operatorname{Dual}\left(\operatorname{Out}\left(\operatorname{Dual}\left([a, b]^{*}\right)\right)\right) .
$$

So the implementation of the inner representation can be realized by the outer representation. Furthermore, $[a, b]^{*} \subseteq \operatorname{Out}\left([a, b]^{*}\right)$, which is to be further applied in the computation of a modal interval inclusion function via modal interval arithmetic.

\subsection{The function of modal intervals - semantic extension}

Comparative to the interval function $f(\mathbf{X})=\left[\min _{\mathbf{x} \in \mathbf{X}} f(\mathbf{x}), \max _{\mathbf{x} \in \mathbf{X}} f(\mathbf{x})\right], \mathbf{X} \in \mathbb{I}\left(\mathbb{R}^{n}\right)$ defined in classical interval analysis, the modal interval function $f^{*}\left(\mathbf{X}^{*}\right): \mathbb{I}^{*}\left(\mathbb{R}^{n}\right) \rightarrow \mathbb{I}^{*}(\mathbb{R})$ is defined to be [5]:

$$
f^{*}\left(\mathbf{X}^{*}\right):=\left[\min _{\mathbf{x}_{p} \in \operatorname{Prop}\left(\mathbf{X}_{p}^{*}\right)} \max _{\mathbf{x}_{i} \in \operatorname{Prop}\left(\mathbf{X}_{i}^{*}\right)} f\left(\mathbf{x}_{p}, \mathbf{x}_{i}\right), \max _{\mathbf{x}_{p} \in \operatorname{Prop}\left(\mathbf{X}_{p}^{*}\right)} \min _{\mathbf{x}_{i} \in \operatorname{Prop}\left(\mathbf{X}_{i}^{*}\right)} f\left(\mathbf{x}_{p}, \mathbf{x}_{i}\right)\right]^{*} .
$$

Naturally, $f^{*}\left(\mathbf{X}^{*}\right)$ degenerates to $\left[\min _{\mathbf{x}_{p} \in \operatorname{Prop}\left(\mathbf{X}_{p}^{*}\right)} f\left(\mathbf{x}_{p}\right), \max _{\mathbf{x}_{p} \in \operatorname{Prop}\left(\mathbf{X}_{p}^{*}\right)} f\left(\mathbf{x}_{p}\right)\right]^{*}$ when all components of $\mathbf{X}^{*}$ are proper intervals, which is the case of $f(\mathbf{X})$ in classical interval analysis.

The semantic statement (3.2) for $f(\mathbf{X}) \subseteq F(\mathbf{X})$ in classical interval analysis can be extended accordingly for $f^{*}\left(\mathbf{X}^{*}\right) \subseteq F^{*}\left(\mathbf{X}^{*}\right)$ in modal interval analysis, where $F^{*}\left(\mathbf{X}^{*}\right)$ is a modal interval inclusion function of $f^{*}\left(\mathbf{X}^{*}\right)$ and its computation via modal interval arithmetic is to be addressed in the following subsection. The extended semantic statement for $f^{*}\left(\mathbf{X}^{*}\right) \subseteq F^{*}\left(\mathbf{X}^{*}\right)$ is $[5]$ :

$$
f^{*}\left(\mathbf{X}^{*}\right) \subseteq F^{*}\left(\mathbf{X}^{*}\right) \Leftrightarrow \forall \mathbf{x}_{p} \in \operatorname{Prop}\left(\mathbf{X}_{p}^{*}\right) \mathbf{Q}\left(F^{*}\left(\mathbf{X}^{*}\right)\right) z \in \operatorname{Prop}\left(F^{*}\left(\mathbf{X}^{*}\right)\right) \exists \mathbf{x}_{i} \in \operatorname{Prop}\left(\mathbf{X}_{i}^{*}\right) z=f\left(\mathbf{x}_{p}, \mathbf{x}_{i}\right)
$$

This is the fundamental semantic theorem in modal interval analysis because it provides a semantic interpretation for the relationship between the modal interval function $f^{*}\left(\mathbf{X}^{*}\right)$ and its inclusion function $F^{*}\left(\mathbf{X}^{*}\right)$. It is worthy to note that the modality of $F^{*}\left(\mathbf{X}^{*}\right)$ depends on its bounds and the order of quantifiers for function variables is fixed with respect to their modalities in the extended semantic statement. The semantic 
theorem can be derived as follows [5]: $f^{*}\left(\mathbf{X}^{*}\right) \subseteq F^{*}\left(\mathbf{X}^{*}\right) \Leftrightarrow \forall \mathbf{x}_{p} \in \operatorname{Prop}\left(\mathbf{X}_{p}^{*}\right) f^{*}\left(\mathbf{x}_{p}, \mathbf{X}_{i}^{*}\right) \subseteq F^{*}\left(\mathbf{X}^{*}\right) \Leftrightarrow \forall \mathbf{x}_{p} \in$ $\operatorname{Prop}\left(\mathbf{X}_{p}^{*}\right)\left\{\begin{array}{l}\text { if } F^{*}\left(\mathbf{X}^{*}\right) \text { is proper, then } \operatorname{Prop}\left(f^{*}\left(\mathbf{x}_{p}, \mathbf{X}_{i}^{*}\right)\right) \cap \operatorname{Prop}\left(F^{*}\left(\mathbf{X}^{*}\right)\right) \neq \emptyset \\ \text { if } F^{*}\left(\mathbf{X}^{*}\right) \text { is improper, then } \operatorname{Prop}\left(F^{*}\left(\mathbf{X}^{*}\right)\right) \subseteq \operatorname{Prop}\left(f^{*}\left(\mathbf{x}_{p}, \mathbf{X}_{i}^{*}\right)\right)\end{array} \Leftrightarrow \forall \mathbf{x}_{p} \in \operatorname{Prop}\left(\mathbf{X}_{p}^{*}\right) \mathbf{Q}\left(F^{*}\left(\mathbf{X}^{*}\right)\right) z\right.$ $\in \operatorname{Prop}\left(F^{*}\left(\mathbf{X}^{*}\right)\right) z \in \operatorname{Prop}\left(f^{*}\left(\mathbf{x}_{p}, \mathbf{X}_{i}^{*}\right)\right) \Leftrightarrow \forall \mathbf{x}_{p} \in \operatorname{Prop}\left(\mathbf{X}_{p}^{*}\right) \mathbf{Q}\left(F^{*}\left(\mathbf{X}^{*}\right)\right) z \in \operatorname{Prop}\left(F^{*}\left(\mathbf{X}^{*}\right)\right) \exists \mathbf{x}_{i} \in \operatorname{Prop}\left(\mathbf{X}_{i}^{*}\right) z=$ $f\left(\mathbf{x}_{p}, \mathbf{x}_{i}\right)$.

It can be seen that the extended semantic statement (3.14) in modal interval analysis introduces both the quantifier $\forall \mathbf{x}_{p} \in \operatorname{Prop}\left(\mathbf{X}_{p}^{*}\right)$ and the quantifier $\exists \mathbf{x}_{i} \in \operatorname{Prop}\left(\mathbf{X}_{i}^{*}\right)$ for function variables while the semantic statement (3.2) in classical interval analysis only contains the quantifier $\forall \mathbf{x} \in \mathbf{X}$ for all function variables. Such a semantic extension is essential and complete in theory because many physical problems include both $\forall$ and $\exists$ for function variables, just as the definition of one-step robust controllable sets in (2.2). Oppositely, the semantic statement for $f^{*}\left(\mathbf{X}^{*}\right) \nsubseteq G^{*}\left(\mathbf{X}^{*}\right)$ is as follows:

$$
f^{*}\left(\mathbf{X}^{*}\right) \nsubseteq G^{*}\left(\mathbf{X}^{*}\right) \Leftrightarrow \exists \mathbf{x}_{p} \in \operatorname{Prop}\left(\mathbf{X}_{p}^{*}\right) \mathbf{Q}\left(\operatorname{Dual}\left(G^{*}\left(\mathbf{X}^{*}\right)\right)\right) z \in \operatorname{Prop}\left(G^{*}\left(\mathbf{X}^{*}\right)\right) \forall \mathbf{x}_{i} \in \operatorname{Prop}\left(\mathbf{X}_{i}^{*}\right) z \neq f\left(\mathbf{x}_{p}, \mathbf{x}_{i}\right)
$$

\subsection{The computation of $\boldsymbol{F}^{*}\left(\mathrm{X}^{*}\right)$ - arithmetic extension}

Similar to the interval function $f(\mathbf{X})$ in classical interval analysis, the modal interval function $f^{*}\left(\mathbf{X}^{*}\right)$ can seldom be computed arithmetically as its bounds concern complex minimax and maximin optimization problems. The inclusion function $F(\mathbf{X})$ computed directly through interval arithmetic is used instead in various solvers of classical interval analysis to provide the lower and upper bounds of the original interval function $f(\mathbf{X})$. Such a strategy can be extended to the approximation of the modal interval function $f^{*}\left(\mathbf{X}^{*}\right)$ as well. Modal interval arithmetic is defined to compute an outer approximation $f^{*}\left(\mathbf{X}^{*}\right) \subseteq$ Outer $\left(f^{*}\left(\mathbf{X}^{*}\right)\right)$ as well as an inner approximation $\operatorname{Inner}\left(f^{*}\left(\mathbf{X}^{*}\right)\right) \subseteq f^{*}\left(\mathbf{X}^{*}\right)$ arithmetically and directly while the semantic theorem (3.14) is applied to interpret such approximations $[5,7]$. The outer approximation Outer $\left(f^{*}\left(\mathbf{X}^{*}\right)\right)$ of $f^{*}\left(\mathbf{X}^{*}\right)$ can also be regarded as a modal interval inclusion function $F^{*}\left(\mathbf{X}^{*}\right)$ of $f^{*}\left(\mathbf{X}^{*}\right)$. In the following paragraphs, the arithmetic extension for computing the modal interval inclusion function $F^{*}\left(\mathbf{X}^{*}\right)$ of $f^{*}\left(\mathbf{X}^{*}\right)$ is to be explained in detail.

Four elementary arithmetic operations $(+,-, \times, \div)$ between two modal intervals $[a, b]^{*}$ and $[c, d]^{*}$ can be computed according to the definition (3.13), i.e., $f^{*}\left([a, b]^{*},[c, d]^{*}\right)=[a, b]^{*} \diamond[c, d]^{*}(\diamond=+,-, \times, \div)$, where the outer representation of a modal interval is used whenever necessary. For instance, $[3,1]^{*}+[2,3]^{*}=[5,4]^{*}$ and $[3,1]^{*}-[2,3]^{*}=[0,-1]^{*}$. It turns out that the arithmetic extension coincides with Kaucher's complete arithmetic for classical intervals [9]. Such an arithmetic is adopted as modal interval arithmetic and the semantic theorem (3.14) is maintained to interpret the computation results.

Similarly to four elementary arithmetic operations, other operators between modal intervals or one-variable operators for a modal interval can be computed according to the definition (3.13) as well. A general modal rational function can also be computed by decomposing it into basic operations according to the syntactic tree of the expression defining the function once all basic operators have been defined according to the definition (3.13), which is similar to the computation of natural inclusion functions in classical interval analysis. Concretely, the modal rational *-extension function $f R^{*}\left(\mathbf{X}^{*}\right)$ is defined to be the function from $\mathbb{I}^{*}\left(\mathbb{R}^{n}\right)$ to $\mathbb{I}^{*}(\mathbb{R})$ determined by the syntactic tree of the expression defining the function when all the operators concerned are computed according to the definition (3.13). The obtained $f R^{*}\left(\mathbf{X}^{*}\right)$ can also be referred to as the natural modal interval inclusion function for $f^{*}\left(\mathbf{X}^{*}\right)$. Then the generally uncomputable $f^{*}\left(\mathbf{X}^{*}\right)$ can be approximated by the arithmetically computable modal interval inclusion function $F^{*}\left(\mathbf{X}^{*}\right)$. Taking $f\left(x_{1}, x_{2}\right)=x_{1} x_{2}+\left(x_{1}+x_{2}\right)^{2}$ as an example $[5], f R^{*}\left([-1,1]^{*},[1,-1]^{*}\right)=[0,0]^{*}+[1,0]^{*}=[1,0]^{*}$ and thus $f^{*}\left([-1,1]^{*},[1,-1]^{*}\right) \subseteq[1,0]^{*}$.

\subsection{The solver of quantified set inversion}

In contrast to the computation of $f^{*}\left(\mathbf{X}^{*}\right)$ with the known domain $\mathbf{X}^{*}$, the opposite problem is finding the feasible solution in $\mathbf{X}^{*}$ with the known range $[a, b]^{*}$, i.e., to find all $\mathcal{X}^{*}=\left(\mathcal{X}_{p}^{*}, \mathcal{X}_{i}^{*}\right)$ within the initial domain $\mathbf{X}^{*}$ that satisfies

$$
\forall \mathbf{x}_{p} \in \operatorname{Prop}\left(\mathcal{X}_{p}^{*}\right) \mathbf{Q}\left([a, b]^{*}\right) z \in \operatorname{Prop}\left([a, b]^{*}\right) \exists \mathbf{x}_{i} \in \operatorname{Prop}\left(\mathcal{X}_{i}^{*}\right) z=f\left(\mathbf{x}_{p}, \mathbf{x}_{i}\right)
$$


TABLE 1. 1-Dimensional Quantified Set Inversion (1-D QST).

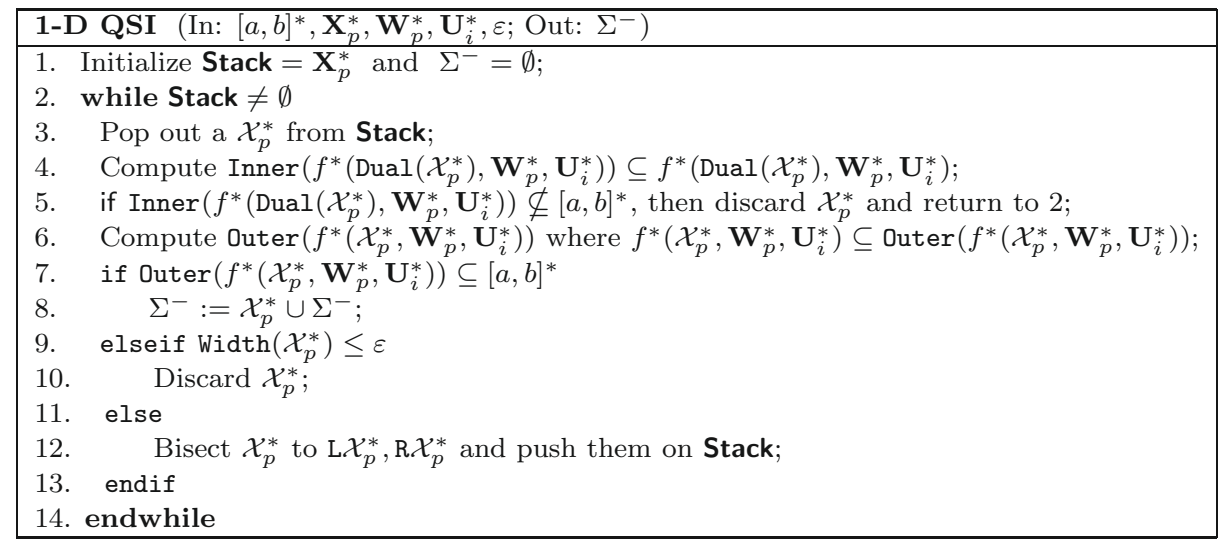

When the modality of all components of $\mathbf{X}^{*}$ is $\exists$, this problem degenerates to be a traditional set inversion problem discussed in classical interval analysis; when the modality of all components of $\mathbf{X}^{*}$ is not uniformly $\exists$, this problem generalizes to be a quantified set inversion problem discussed in modal interval analysis [7], where only the solver of 1-dimensional quantified set inversion was proposed. Many physical problems can be formulated mathematically as a quantified constraint satisfaction problem, which may be solved with the solver of quantified set inversion $[7,17]$. For example, to find the one-step robust controllable set $\tilde{\mathcal{K}}(\mathbf{X}, \mathbb{T})$ defined in $(2.2)$ is a typical quantified constraint satisfaction problem and then $\tilde{\mathcal{K}}(\mathbf{X}, \mathbb{T})$ can be obtained numerically via the solver of quantified set inversion. Concretely, the problem of finding the subset $\Sigma_{\mathbf{x}} \subseteq \operatorname{Prop}\left(\mathbf{X}^{*}\right) \subseteq \mathbb{I}\left(\mathbb{R}^{n}\right)$ within which there exists a control input $\mathbf{u} \in \operatorname{Prop}\left(\mathbf{U}^{*}\right) \subseteq \mathbb{I}\left(\mathbb{R}^{m}\right)$ to render the scalar function $f(\mathbf{x}, \mathbf{w}, \mathbf{u}) \in \operatorname{Prop}\left([a, b]^{*}\right)$ for all uncertain cases $\mathbf{w} \in \operatorname{Prop}\left(\mathbf{W}^{*}\right) \subseteq \mathbb{I}\left(\mathbb{R}^{l}\right)$ can be solved using the solver of 1-dimensional quantified set inversion with the function $f^{*}\left(\mathbf{X}_{p}^{*}, \mathbf{W}_{p}^{*}, \mathbf{U}_{i}^{*}\right)$, where $\mathbf{X}_{p}^{*}$ is a proper interval vector with $\mathbf{X}_{p}^{*}=\mathbf{X}^{*}, \mathbf{W}_{p}^{*}$ is also a proper interval vector with $\mathbf{W}_{p}^{*}=\mathbf{W}^{*}, \mathbf{U}_{i}^{*}$ is an improper interval vector with $\mathbf{U}_{i}^{*}=\mathbf{U}^{*}$ and the scalar function $f(\mathbf{x}, \mathbf{w}, \mathbf{u})$ is for describing the selected terminal set $\mathbb{T}$. The solver of 1-dimensional quantified set inversion for finding the inner approximation $\Sigma^{-}$of $\Sigma_{\mathbf{x}}$ is illustrated in Table 1, where $\varepsilon$ is the bound of tolerance for an interval beyond which the interval is not to be bisected further.

As shown in Table $1, \mathbf{X}_{p}^{*}$ is the proper modal interval vector for the initial state space; $\mathbf{U}_{i}^{*}$ is the improper modal interval vector for the admissible control space; and $\Sigma^{-}$is to store the inner approximation of the onestep robust controllable set. The inner approximation of $f^{*}\left(\operatorname{Dual}\left(\mathcal{X}_{p}^{*}\right), \mathbf{W}_{p}^{*}, \mathbf{U}_{i}^{*}\right)$ and the outer approximation of $f^{*}\left(\mathcal{X}_{p}^{*}, \mathbf{W}_{p}^{*}, \mathbf{U}_{i}^{*}\right)$ are computed in Step 4 and Step 6 , respectively; if Inner $\left(f^{*}\left(\operatorname{Dual}\left(\mathcal{X}_{p}^{*}\right), \mathbf{W}_{p}^{*}, \mathbf{U}_{i}^{*}\right)\right) \nsubseteq[a, b]^{*}$, then $f^{*}\left(\operatorname{Dual}\left(\mathcal{X}_{p}^{*}\right), \mathbf{W}_{p}^{*}, \mathbf{U}_{i}^{*}\right) \nsubseteq[a, b]^{*}$ as well, which signifies $\exists \mathbf{w} \in \operatorname{Prop}\left(\mathbf{W}_{p}^{*}\right) \mathbf{Q}\left([b, a]^{*}\right) z \in \operatorname{Prop}\left([a, b]^{*}\right) \forall \mathbf{x} \in$ $\operatorname{Prop}\left(\mathcal{X}_{p}^{*}\right) \forall \mathbf{u} \in \operatorname{Prop}\left(\mathbf{U}_{i}^{*}\right) z \neq f(\mathbf{x}, \mathbf{w}, \mathbf{u})$ according to the semantic statement (3.15), thus $\mathcal{X}_{p}^{*}$ cannot belong to the one-step robust controllable set and it is to be discarded in Step 5; if Outer $\left(f^{*}\left(\mathcal{X}_{p}^{*}, \mathbf{W}_{p}^{*}, \mathbf{U}_{i}^{*}\right)\right) \subseteq[a, b]^{*}$, then $f^{*}\left(\mathcal{X}_{p}^{*}, \mathbf{W}_{p}^{*}, \mathbf{U}_{i}^{*}\right) \subseteq[a, b]^{*}$, which signifies $\forall \mathbf{x} \in \operatorname{Prop}\left(\mathcal{X}_{p}^{*}\right) \forall \mathbf{w} \in \operatorname{Prop}\left(\mathbf{W}_{p}^{*}\right) \mathbf{Q}\left([a, b]^{*}\right) z \in \operatorname{Prop}\left([a, b]^{*}\right) \exists \mathbf{u} \in$ $\operatorname{Prop}\left(\mathbf{U}_{i}^{*}\right) z=f(\mathbf{x}, \mathbf{w}, \mathbf{u})$ according to the semantic statement (3.14), thus $\mathcal{X}_{p}^{*}$ belongs to the one-step robust controllable set and it is to be added into the inner approximation $\Sigma^{-}$in Step 8; if no judgment can be made in either Step 5 or Step 7, and the width of the modal interval vector $\mathcal{X}_{p}^{*}$ is not bigger than the bound of tolerance $\varepsilon$, it is to be discarded as well in Step 10; and finally, if no judgment can be made in either Step 5 or Step 7, and $\operatorname{Width}\left(\mathcal{X}_{p}^{*}\right)$ is bigger than the bound of tolerance $\varepsilon$, it is to be bisected into two sub-modal interval vectors $\mathrm{L} \mathcal{X}_{p}^{*}, \mathrm{R} \mathcal{X}_{p}^{*}$ in Step 12 and both of them are to be pushed on Stack for further judgments. It should be emphasized that the modality of $\mathcal{X}_{p}^{*}$ has to be changed using the operator Dual so as to use the negative semantic statement (3.15) to discard it contrary to use the affirmative semantic statement (3.14) to accept it as a part of the one-step robust controllable set. 
TABLe 2. 2-Dimensional Quantified Set Inversion (2-D QST).

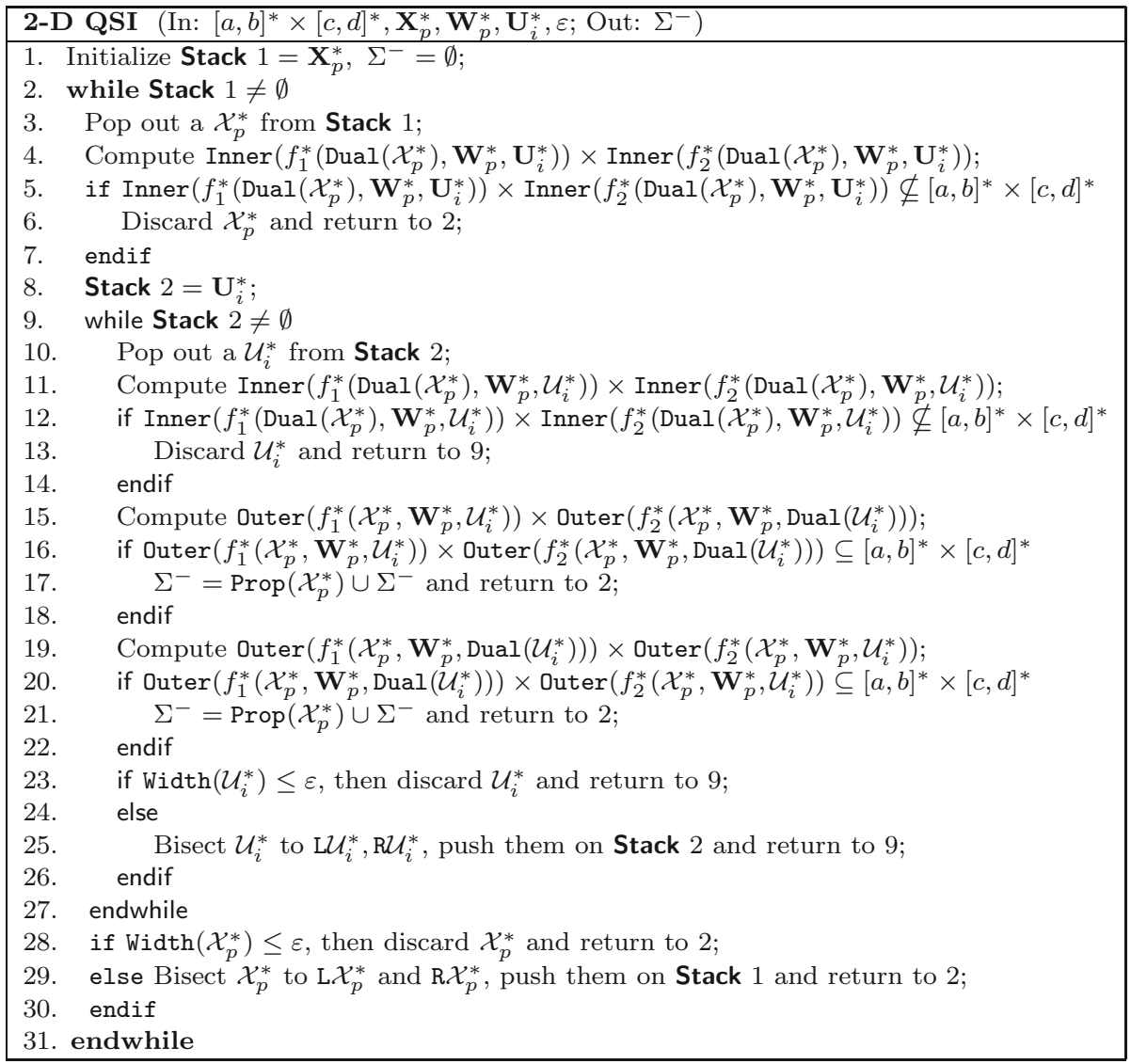

The solver of 1 -dimensional quantified set inversion in Table 1 can be extended to $\mathbf{f}^{*}\left(\mathbf{X}^{*}\right): \mathbb{I}^{*}\left(\mathbb{R}^{n}\right) \rightarrow \mathbb{I}^{*}\left(\mathbb{R}^{m}\right)$, where inclusion and exclusion judgments between modal interval vectors are encountered and corresponding semantic statements are derived from the combination of several affirmative semantic statements or several negative semantic statements. For instance, assume that $\mathbf{f}(x)=f_{1}(x) \times f_{2}(x)$, where $f_{1}\left([1,2]^{*}\right) \subseteq[3,4]^{*}$ and $f_{2}\left(\operatorname{Dual}\left([1,2]^{*}\right)\right) \subseteq[4,5]^{*}$, then the overall semantic statement is $\exists z_{1} \in[3,4] \exists z_{2} \in[4,5] \exists x \in[1,2]$ $z_{1}=f_{1}(x), z_{2}=f_{2}(x)$ according to the combination of two affirmative semantic statements $\forall x \in[1,2] \exists z_{1} \in$ $[3,4] z_{1}=f_{1}(x)$ and $\exists z_{2} \in[4,5] \exists x \in[1,2] z_{2}=f_{2}(x)$. Taking the 2-dimensional quantified set inversion as an example, the known range of the 2 -dimensional function vector $\mathbf{f}(\mathbf{x}, \mathbf{w}, \mathbf{u})=f_{1}(\mathbf{x}, \mathbf{w}, \mathbf{u}) \times f_{2}(\mathbf{x}, \mathbf{w}, \mathbf{u})$ is assumed to be $[a, b]^{*} \times[c, d]^{*}$. The problem is still to find the subset $\Sigma_{\mathbf{x}} \subseteq \operatorname{Prop}\left(\mathbf{X}^{*}\right) \subseteq \mathbb{I}\left(\mathbb{R}^{n}\right)$ within which there exists a control input $\mathbf{u} \in \operatorname{Prop}\left(\mathbf{U}^{*}\right) \subseteq \mathbb{I}\left(\mathbb{R}^{m}\right)$ to render the 2-dimensional function vector $\mathbf{f}(\mathbf{x}, \mathbf{w}, \mathbf{u}) \in \operatorname{Prop}\left([a, b]^{*}\right) \times$ $\operatorname{Prop}\left([c, d]^{*}\right)$ for all uncertain cases $\mathbf{w} \in \operatorname{Prop}\left(\mathbf{W}^{*}\right) \subseteq \mathbb{I}\left(\mathbb{R}^{l}\right)$. The extended solver of 2-dimensional quantified set inversion for finding the inner approximation $\Sigma^{-}$of $\Sigma_{\mathbf{x}}$ is illustrated in Table 2 , where $\mathbf{X}_{p}^{*}$ is for the initial state space and $\mathbf{U}_{i}^{*}$ is for the admissible control space. Since the combined affirmative semantic statement for accepting $\mathcal{X}_{p}^{*}$ as a part of $\Sigma_{\mathbf{x}}$ is a combination of the semantic statement for $f_{1}^{*} \subseteq[a, b]^{*}$ and the semantic statement for $f_{2}^{*} \subseteq[c, d]^{*}$, thus $\operatorname{Dual}\left(\mathcal{U}_{i}^{*}\right)$ should occur in one of $f_{1}^{*}$ and $f_{2}^{*}$ to change the semantic statement (3.14) for ensuring that there exists the same $\mathbf{u} \in \operatorname{Prop}\left(\mathcal{U}_{i}^{*}\right)$ to render $f_{1}^{*} \times f_{2}^{*} \subseteq[a, b]^{*} \times[c, d]^{*}$ in $\operatorname{Step} 16$ and Step 20, i.e., $\exists \mathbf{u} \in \operatorname{Prop}\left(\mathcal{U}_{i}^{*}\right) f_{1}^{*} \subseteq[a, b]^{*}, \forall \mathbf{u} \in \operatorname{Prop}\left(\operatorname{Dual}\left(\mathcal{U}_{i}^{*}\right)\right) f_{2}^{*} \subseteq[c, d]^{*}$ or $\forall \mathbf{u} \in \operatorname{Prop}\left(\operatorname{Dual}\left(\mathcal{U}_{i}^{*}\right)\right) f_{1}^{*} \subseteq[a, b]^{*}$, $\exists \mathbf{u} \in \operatorname{Prop}\left(\mathcal{U}_{i}^{*}\right) f_{2}^{*} \subseteq[c, d]^{*}$ leads to $\exists \mathbf{u} \in \operatorname{Prop}\left(\mathcal{U}_{i}^{*}\right) f_{1}^{*} \times f_{2}^{*} \subseteq[a, b]^{*} \times[c, d]^{*}$ while $\exists \mathbf{u} \in \operatorname{Prop}\left(\mathcal{U}_{i}^{*}\right) f_{1}^{*} \subseteq[a, b]^{*}$, $\exists \mathbf{u} \in \operatorname{Prop}\left(\mathcal{U}_{i}^{*}\right) f_{2}^{*} \subseteq[c, d]^{*}$ cannot lead to $\exists \mathbf{u} \in \operatorname{Prop}\left(\mathcal{U}_{i}^{*}\right) f_{1}^{*} \times f_{2}^{*} \subseteq[a, b]^{*} \times[c, d]^{*}$ because there might not exist 
the same $\mathbf{u} \in \operatorname{Prop}\left(\mathcal{U}_{i}^{*}\right)$ to satisfy $f_{1}^{*} \subseteq[a, b]^{*}$ and $f_{2}^{*} \subseteq[c, d]^{*}$ simultaneously. To require all $\mathbf{u} \in \operatorname{Prop}\left(\mathcal{U}_{i}^{*}\right)$ to satisfy $f_{1}^{*}\left(\mathcal{X}_{p}^{*}, \mathbf{W}_{p}^{*}, \operatorname{Dual}\left(\mathcal{U}_{i}^{*}\right)\right) \subseteq[a, b]^{*}$ or $f_{2}^{*}\left(\mathcal{X}_{p}^{*}, \mathbf{W}_{p}^{*}, \operatorname{Dual}\left(\mathcal{U}_{i}^{*}\right)\right) \subseteq[c, d]^{*}$ is strict and thus $\mathcal{U}_{i}^{*}$ needs to be bisected in Step 25. Since $f_{1}^{*} \times f_{2}^{*} \nsubseteq[a, b]^{*} \times[c, d]^{*}$ is equal to $f_{1}^{*} \nsubseteq[a, b]^{*}$ or $f_{2}^{*} \nsubseteq[c, d]^{*}$, then the combined negative semantic statement for discarding modal interval vectors is a direct combination of the semantic statement for $f_{1}^{*} \nsubseteq[a, b]^{*}$ and the semantic statement for $f_{2}^{*} \nsubseteq[c, d]^{*}$. However, the entire domain of $\mathbf{U}_{i}^{*}$ is tested in Step 5 and thus the corresponding $\mathcal{X}_{p}^{*}$ is to be discarded in Step 6 while only a part of $\mathbf{U}_{i}^{*}$ is tested in Step 12 and thus only that part of $\mathbf{U}_{i}^{*}$ is to be discarded in Step 13. It is worthy to note that the principle and the structure of multi-dimensional quantified set inversion is similar to the 2-dimensional case while only inclusion and exclusion judgments are performed between higher-dimensional modal interval vectors.

\subsection{The solver of constrained minimax optimization}

A general constrained minimax optimization problem can be formulated as:

$$
\min _{\mathbf{u} \in \mathbf{U}_{0}} \max _{\mathbf{w} \in \mathbf{W}_{0}} f(\mathbf{w}, \mathbf{u}), \forall \mathbf{w} \in \mathbf{W}_{0} \exists \mathbf{u} \in \mathbf{U}_{0}: \mathbf{g}(\mathbf{w}, \mathbf{u}) \in \mathbb{C},
$$

where $\mathbf{w} \in \mathbf{W}_{0}$ is usually referred to as uncertain parameters or additive disturbances and $\mathbf{u} \in \mathbf{U}_{0}$ is usually referred to as decision variables; and then $\forall \mathbf{w} \in \mathbf{W}_{0} \exists \mathbf{u} \in \mathbf{U}_{0}: \mathbf{g}(\mathbf{w}, \mathbf{u}) \in \mathbb{C}$ is actually a constraint imposed only on $\mathbf{u}$. Various application problems concerning control and decision can be cast in the framework of constrained minimax optimizations, just as the computation of one-step control inputs in (2.4). According to the definition of $f^{*}$ in (3.13), the minimax value of $\min _{\mathbf{u} \in \mathbf{U}_{0}} \max _{\mathbf{w} \in \mathbf{W}_{0}} f(\mathbf{w}, \mathbf{u})$ is equal to $\operatorname{Lb}\left(f^{*}\left(\mathbf{X}_{p}^{*}, \mathbf{X}_{i}^{*}\right)\right)$, where $\mathbf{X}_{p}^{*}=\mathbf{U}^{*}$, $\mathbf{X}_{i}^{*}=\mathbf{W}^{*}$ and $\operatorname{Prop}\left(\mathbf{U}^{*}\right)=\mathbf{U}_{0}, \operatorname{Prop}\left(\mathbf{W}^{*}\right)=\mathbf{W}_{0}$. The constrained minimax optimization problem (3.17) can thus be transformed to the approximation of the lower bound of $f^{*}\left(\mathbf{X}_{p}^{*}, \mathbf{X}_{i}^{*}\right)$. Furthermore, it is necessary to find the exact feasible solution $\Sigma_{\mathbf{u}}$ of the imposed constraint, i.e., $\Sigma_{\mathbf{u}}=\left\{u \mid \forall \mathbf{w} \in \mathbf{W}_{0} \forall \mathbf{u} \in \Sigma_{\mathbf{U}}: \mathbf{g}(\mathbf{w}, \mathbf{u}) \in \mathbb{C}\right\}$, rather than only to determine the existence of $\mathbf{u}$ satisfying $\forall \mathbf{w} \in \mathbf{W}_{0} \exists \mathbf{u} \in \mathbf{U}_{0}: \mathbf{g}(\mathbf{w}, \mathbf{u}) \in \mathbb{C}$ for the purpose of further practical utilization such as obtaining the concrete control input except for only knowing its existence. So the solver of traditional set inversion via interval analysis should be applied to obtain this feasible solution $\Sigma_{\mathbf{u}}$ in advance and then to search the optimal one among all candidates in the feasible solution. The detail of the proposed solver of constrained minimax optimization is illustrated in Table 3, where $\Omega$ stores all feasible subboxes of $\mathbf{U}_{0}$ for the constrained minimax optimization (3.17) and the bound of tolerance $\varepsilon$ for intervals is selected to be small enough so as to obtain a non-empty inner approximation $\Sigma^{-}$of the feasible solution $\Sigma_{\mathbf{u}}$.

As shown in Table 3, an inner approximation $\Sigma^{-}$of the feasible solution $\Sigma_{\mathbf{u}}$ for the imposed constraint $\forall \mathbf{w} \in \mathbf{W}_{0} \forall \mathbf{u} \in \Sigma_{\mathbf{u}}: \mathbf{g}(\mathbf{w}, \mathbf{u}) \in \mathbb{C}$ is obtained using the solver of traditional set inversion via interval analysis from Step 1 to Step 10; then an inclusion domain $[a, b]$ for the constrained minimax function, i.e., $\min _{\mathbf{u} \in \Sigma_{\mathbf{u}}} \max _{\mathbf{w} \in \mathbf{W}_{0}} f(\mathbf{w}, \mathbf{u}) \in[a, b]$, can be approximated through the process from Step 11 to Step 16, where local points $\mathbf{u} \in \mathbf{U}$ and $\mathbf{w} \in \mathbf{W}_{0}$ are applied to obtain better lower and upper bounds of this constrained minimax function in Step 14 and Step 15 based on the fact that $\min _{\mathbf{u} \in \Sigma_{\mathbf{u}}} \max _{\mathbf{w} \in \mathbf{W}_{0}} f(\mathbf{w}, \mathbf{u}) \geq \min _{\mathbf{u} \in \Sigma_{\mathbf{u}}} f\left(\mathbf{w}_{0}, \mathbf{u}\right), \mathbf{w}_{0} \in$ $\mathbf{W}_{0}$ and $\min _{\mathbf{u} \in \Sigma_{\mathbf{u}}} \max _{\mathbf{w} \in \mathbf{W}_{0}} f(\mathbf{w}, \mathbf{u}) \leq \max _{\mathbf{w} \in \mathbf{W}_{0}} f\left(\mathbf{w}, \mathbf{u}_{0}\right), \mathbf{u}_{0} \in \Sigma_{\mathbf{u}}$, respectively. This is equal to obtain better lower and upper bounds of $\operatorname{Lb}\left(f^{*}\left(\mathbf{X}_{p}^{*}, \mathbf{X}_{i}^{*}\right)\right)$, where $\mathbf{X}_{p}^{*}=\mathbf{U}^{*}, \mathbf{X}_{i}^{*}=\mathbf{W}^{*}$. However, local searches for better points $\mathbf{u} \in \mathbf{U}, \mathbf{w} \in \mathbf{W}_{0}$ and bisections of those subboxes with $\operatorname{Width}(\mathbf{U})>\varepsilon$ can be performed further to obtain a more precise inclusion domain for the constrained minimax function. The obtained inclusion domain is used in Step 17 to find any potential $\mathbf{U}_{m}$ to be stored in $\Omega$ that may result in the overall minimax optimization value. It can be seen that the main feature of the proposed constrained minimax optimization algorithm is to perform the search of the feasible solution and the search of the optimal solution sequentially, which is different from the constrained minimax algorithm addressed in [8] where the test of feasibility and the test of optimality are performed simultaneously. The sequential search of the feasible solution and the optimal solution facilitates the addressed one-step control approach where feasibility implies stability and the amount of efforts allocated for searching the optimal control input can be adjusted according to the practical requirements. 
TABle 3. Constrained Minimax Optimization Algorithm (CMOA).

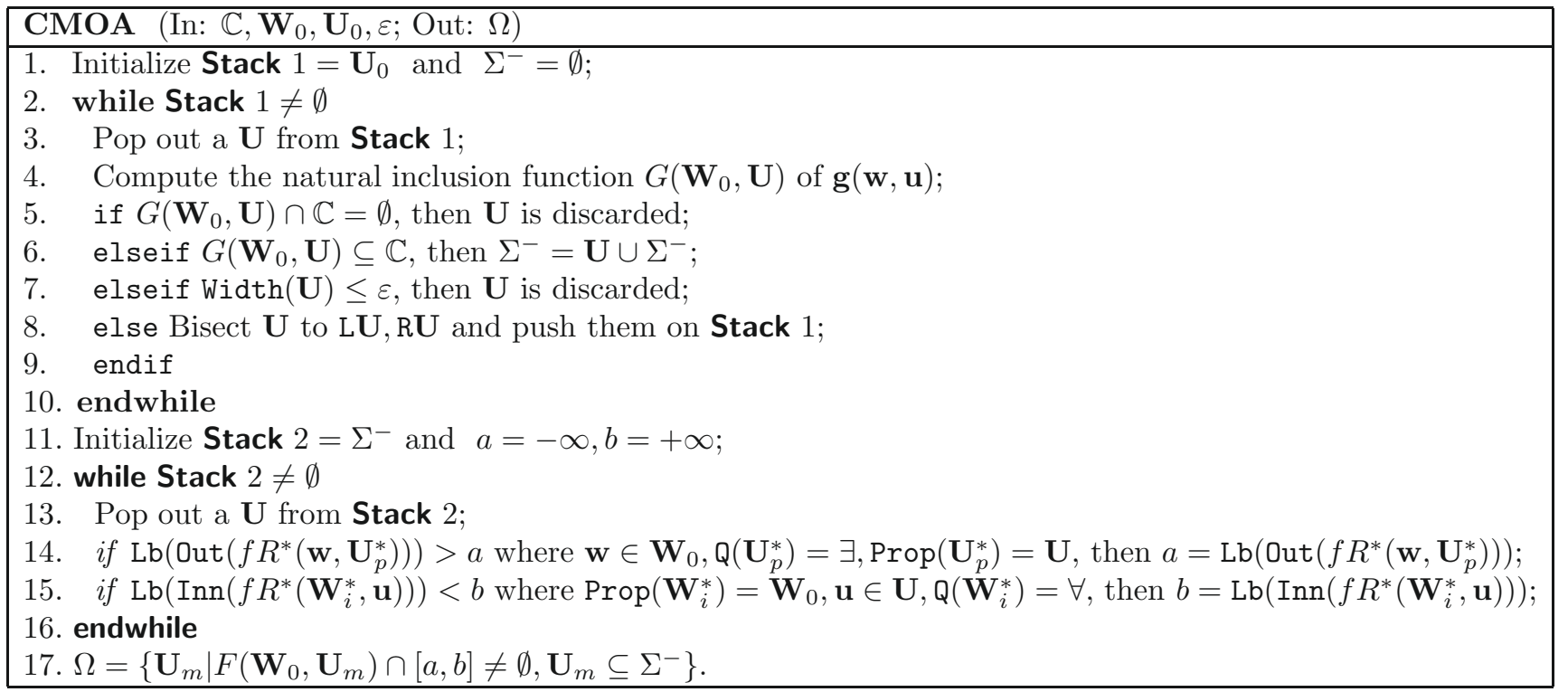

\section{One-STEP CONTROL VIA ROBUSt CONTROLlable SETS}

In this section, the solvers of quantified set inversion and constrained minimax optimization illustrated in Section 3 are applied to solve the problems stated in Section 2, i.e., to compute one-step robust controllable sets and one-step control inputs. The corresponding robust controllable set approximation algorithm, the one-step control algorithm, and feasibility and stability analysis of the addressed one-step control approach are discussed in Sections 4.1, 4.2 and 4.3, respectively.

\subsection{Robust controllable set approximation algorithm}

Assume that the terminal set $\mathbb{T}$ is selected to be a robust control invariant set as well as a polytope, the solver of 1-dimensional quantified set inversion cannot be applied to compute the one-step robust controllable set with a polytope as the terminal set because the inclusion and exclusion judgments between a modal interval vector and a polytope or a union of polytopes are concerned instead. The inclusion and exclusion relationships between a modal interval vector and a polytope or a union of polytopes can be deduced as well according to the principle of inclusion in modal interval analysis. Thus the $i$ th-step robust controllable set $\tilde{\mathcal{K}}_{i}(\mathbf{X}, \mathbb{T})(i=1, \ldots, \infty)$ of a multi-dimensional system with a polytope as the terminal set can also be computed recursively via the solver of multi-dimensional quantified set inversion. The inner approximation of the maximal robust controllable set $\tilde{\mathcal{K}}_{\infty}^{-}(\mathbf{X}, \mathbb{T})$ within the constrained state space is reached when $\tilde{\mathcal{K}}_{N+1}^{-}(\mathbf{X}, \mathbb{T})=\tilde{\mathcal{K}}_{N}^{-}(\mathbf{X}, \mathbb{T})$ for certain $N$ and thus $\tilde{\mathcal{K}}_{\infty}^{-}(\mathbf{X}, \mathbb{T})=\tilde{\mathcal{K}}_{N}^{-}(\mathbf{X}, \mathbb{T})$.

\subsection{One-step control algorithm}

Once the inner approximations $\tilde{\mathcal{K}}_{i}^{-}(\mathbf{X}, \mathbb{T})(i=1, \ldots, N)$ of all the $i$ th-step robust controllable sets within the constrained state space have been obtained via the solver of quantified set inversion, the robust controllability of an initial state can be judged accordingly. Assume that $\mathbf{x}(0) \in \tilde{\mathcal{K}}_{N}^{-}(\mathbf{X}, \mathbb{T})$, i.e., the initial state is robustly controllable to the selected terminal set $\mathbb{T}$ in finite steps, then the one-step control algorithm via a contractive sequence of robust controllable sets is illustrated in Table 4.

According to Table 4, the one-step control algorithm measures the current state in Step 1 and then judges whether the system state has entered into the selected terminal set $\mathbb{T}$ in Step 2 . The related local stabilizing 
TABle 4. One-step Control Algorithm (OCA).

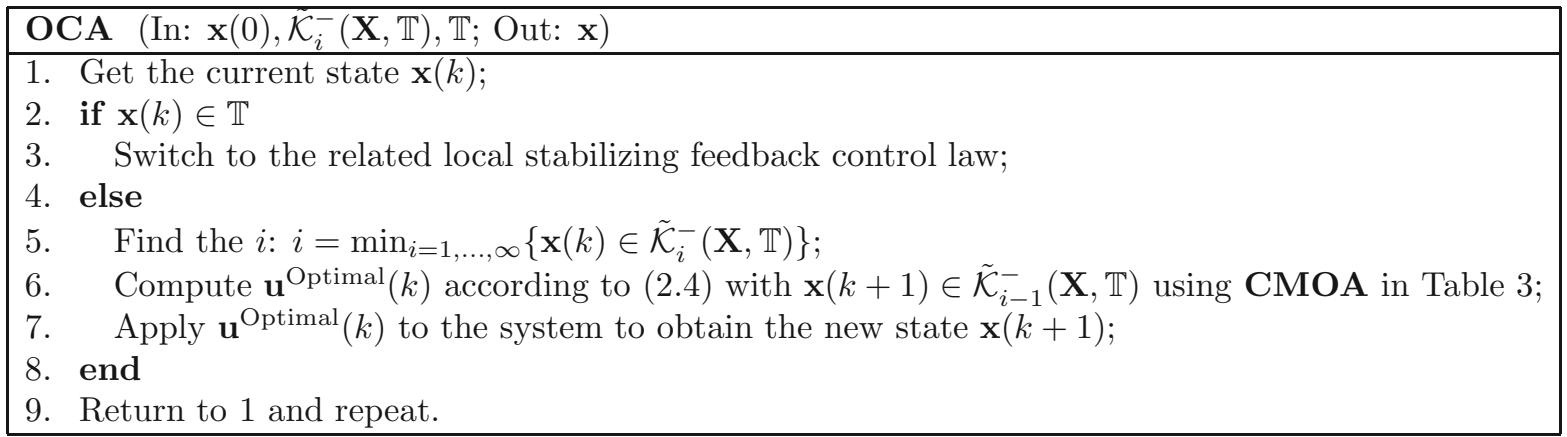

feedback control law is applied if the state has entered into the selected terminal set; otherwise, the algorithm finds the smallest robust controllable set to which the current state belongs in Step 5; the one-step control scheme is formulated according to the strategy of robust model predictive control with unit control horizon in Step 6 and the optimal one-step control input $\mathbf{u}^{\text {Optimal }}(k)$ is computed via the solver of constrained minimax optimization in Table 3. It can be seen from the following feasibility and stability analysis that any feasible solution that satisfies the imposed contractive constraint is an effective control input for the system because such a control input is sufficient to guarantee the stability of the closed-loop system. Thus any feasible control input that satisfies the imposed contractive constraint can be applied as well in the control algorithm to avoid extra efforts for obtaining the exact optimal control input $\mathbf{u}^{\text {Optimal }}(k)$ in Step 6 , which can be easily realized by the proposed constrained minimax optimization algorithm in Table 3.

\subsection{Feasibility and stability analysis}

The feasibility of the addressed one-step control approach means that there always exists a control input $\mathbf{u}$ that can drive the system state belonging to the current robust controllable set $\tilde{\mathcal{K}}_{i}^{-}(\mathbf{X}, \mathbb{T})$ to the next robust controllable set $\tilde{\mathcal{K}}_{i-1}^{-}(\mathbf{X}, \mathbb{T})$. The feasibility of the addressed one-step control approach for a 2-dimensional system is demonstrated in Theorem 4.1 .

Theorem 4.1. For any $\mathbf{x}(k) \in \tilde{\mathcal{K}}_{i}^{-}(\mathbf{X}, \mathbb{T})$, there always exists an admissible control input $\mathbf{u}(k) \in \mathbf{U} \subseteq \mathbb{I}\left(\mathbb{R}^{m}\right)$ that renders $\mathbf{x}(k+1) \in \tilde{\mathcal{K}}_{i-1}^{-}(\mathbf{X}, \mathbb{T}), i=1, \ldots, N$ for all uncertain cases $\mathbf{w}(k) \in \mathbf{W} \subseteq \mathbb{I}\left(\mathbb{R}^{l}\right)$.

Proof. For all robust controllable sets $\tilde{\mathcal{K}}_{i}^{-}(\mathbf{X}, \mathbb{T})(i=1, \ldots, N)$ computed by the solver of 2-dimensional quantified set inversion, if $\operatorname{Prop}\left(\mathbf{X}_{p}^{*}\right) \subseteq \tilde{\mathcal{K}}_{i}^{-}(\mathbf{X}, \mathbb{T})$, then Outer $\left(f_{1}^{*}\left(\mathbf{X}_{p}^{*}, \mathbf{W}_{p}^{*}, \mathbf{U}_{i}^{*}\right)\right) \times \operatorname{Outer}\left(f_{2}^{*}\left(\mathbf{X}_{p}^{*}, \mathbf{W}_{p}^{*}, \operatorname{Dual}\left(\mathbf{U}_{i}^{*}\right)\right)\right) \subseteq$ $\tilde{\mathcal{K}}_{i-1}^{-}(\mathbf{X}, \mathbb{T})$ or Outer $\left(f_{1}^{*}\left(\mathbf{X}_{p}^{*}, \mathbf{W}_{p}^{*}, \operatorname{Dual}\left(\mathbf{U}_{i}^{*}\right)\right)\right) \times \operatorname{Outer}\left(f_{2}^{*}\left(\mathbf{X}_{p}^{*}, \mathbf{W}_{p}^{*}, \mathbf{U}_{i}^{*}\right)\right) \subseteq \tilde{\mathcal{K}}_{i-1}^{-}(\mathbf{X}, \mathbb{T})$ is satisfied, and according to the combination of the underlying two affirmative semantic statements, $\forall \mathbf{x}(k) \in \operatorname{Prop}\left(\mathbf{X}_{p}^{*}\right) \forall \mathbf{w}(k) \in$ $\operatorname{Prop}\left(\mathbf{W}_{p}^{*}\right) \exists \mathbf{x} \in \tilde{\mathcal{K}}_{i-1}^{-}(\mathbf{X}, \mathbb{T}) \exists \mathbf{u}(k) \in \operatorname{Prop}\left(\mathbf{U}_{i}^{*}\right) \mathbf{x}(k+1)=\mathbf{x}$, i.e., the addressed one-step control approach is always feasible.

The initial control invariant set $\mathbb{T}$ is selected to be a robust control invariant set, then $\tilde{\mathcal{K}}_{i-1}(\mathbf{X}, \mathbb{T}) \subseteq \tilde{\mathcal{K}}_{i}(\mathbf{X}, \mathbb{T})$ for all $i=1, \ldots, N$ according to the property of robust control invariant sets [10], i.e., the computed robust controllable sets are inner approximations of a sequence of contractive sets. Thus the feasibility of the addressed one-step control approach leads to stability as the control inputs are to drive the system state contractively along the computed robust controllable sets to the selected terminal robust control invariant set. Once the system state enters the selected terminal control invariant set, the related local stabilizing feedback control law is applied instead to drive the system state to a sufficiently small region around the origin asymptotically. 


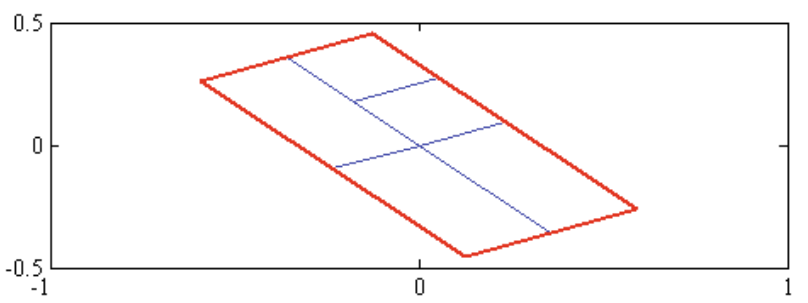

(a) The bisections of the terminal set

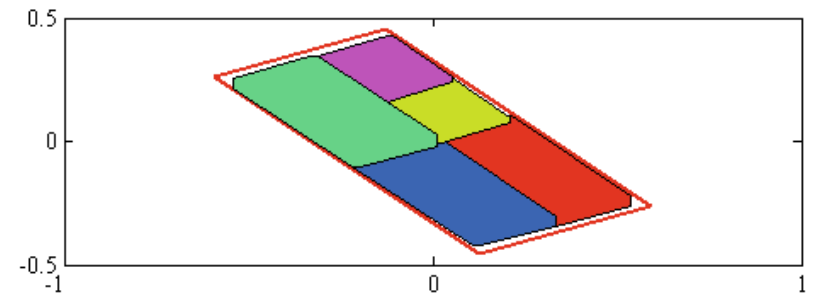

(b) The evolution of every sub-zonotope in the terminal set

Figure 1. The geometrical demonstration of robust control invariance.

\section{An illustrative EXAMPle}

As an illustrative example for demonstrating the addressed one-step control of constrained nonlinear uncertain discrete-time systems via a sequence of robust controllable sets, the system considered is described by the following discrete-time state-space model [3]:

$$
\left\{\begin{array}{l}
x_{1}(k+1)=x_{1}(k)+0.1 x_{2}(k)+0.1\left[\mu(k)+(1-\mu(k)) x_{1}(k)\right] u(k) \\
x_{2}(k+1)=0.1 x_{1}(k)+x_{2}(k)+0.1\left[\mu(k)-4(1-\mu(k)) x_{2}(k)\right] u(k),
\end{array}\right.
$$

where the control input constraint is $|u| \leq 2$ and the states are constrained to $|\mathbf{x}|_{\infty} \leq 2$. The system parameter $\mu$ is assumed to be uncertain here: $\mu \in[0.85,0.95]$. The terminal set $\mathbb{T}$ is selected to be a robust control invariant

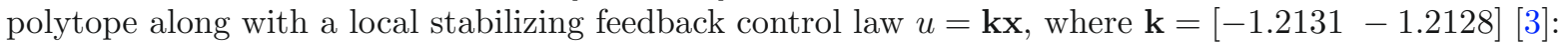

$$
\left[\begin{array}{ll}
0.8190 & -1.9655 \\
-0.8199 & 1.9655 \\
3.0330 & 3.0330 \\
-3.0330 & -3.0330
\end{array}\right] \mathbf{x} \leq\left[\begin{array}{l}
1 \\
1 \\
1 \\
1
\end{array}\right]
$$

The selected terminal polytope $\mathbb{T}$ along with the local stabilizing feedback control law $u=\mathbf{k x}$ can be demonstrated geometrically to be a robust control invariant set by using zonotopes [12], where the selected terminal polytope is bisected into sub-zonotopes and the dynamic evolution of every sub-zonotope under the related local stabilizing feedback control law $u=\mathbf{k x}$ is within the selected terminal polytope $\mathbb{T}$, just as shown in Figure 1 .

The inner approximation of the first-step robust controllable set $\tilde{\mathcal{K}}_{1}(\mathbf{X}, \mathbb{T})$ can be computed using the solver of 2-dimensional quantified set inversion, where the initial terminal set $\mathbb{T}$ is only a polytope. The computed inner approximation of $\tilde{\mathcal{K}}_{1}(\mathbf{X}, \mathbb{T})$ with $\varepsilon=0.05$ is shown in Figure 2, where the computation time on a Pentium Centrino $1.4 \mathrm{GHz}$ Notebook is $4673 \mathrm{~s}$ using MATLAB.

The following-step robust controllable sets can be computed recursively starting from the computed inner approximation of the first-step robust controllable set, where the renewed terminal set for computing the following-step robust controllable set is a union of interval vectors as well as a union of polytopes. The inner approximation of the maximal robust controllable set with $\varepsilon=0.05$ is reached when $\tilde{\mathcal{K}}_{48}^{-}(\mathbf{X}, \mathbb{T})=\tilde{\mathcal{K}}_{49}^{-}(\mathbf{X}, \mathbb{T})$, which is shown in Figure 3. It is worthy to note that all robust controllable sets can be computed offline and the obtained robust controllable sets are to be stored in the computer for the following control simulation. 


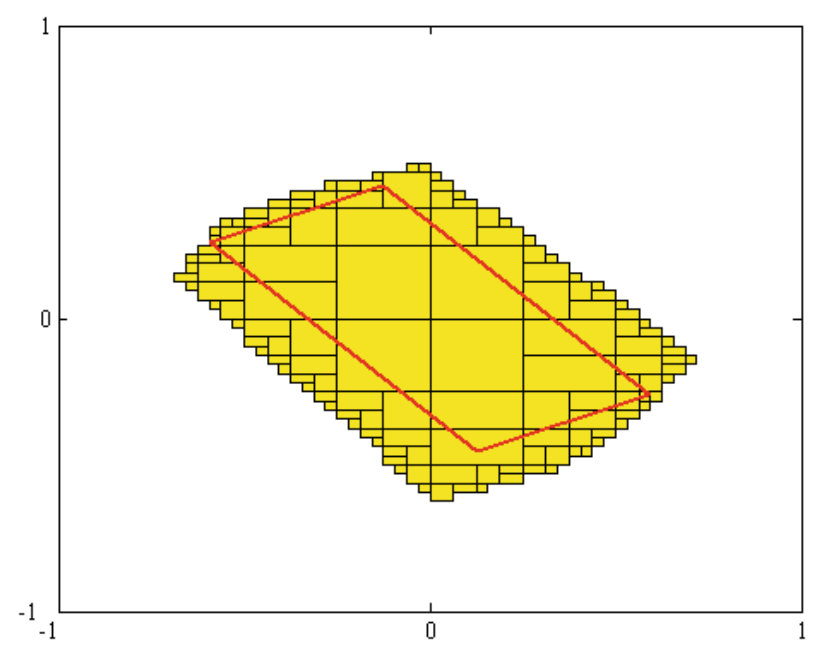

FiguRE 2. The inner approximation of the first-step robust controllable set with $\varepsilon=0.05$.

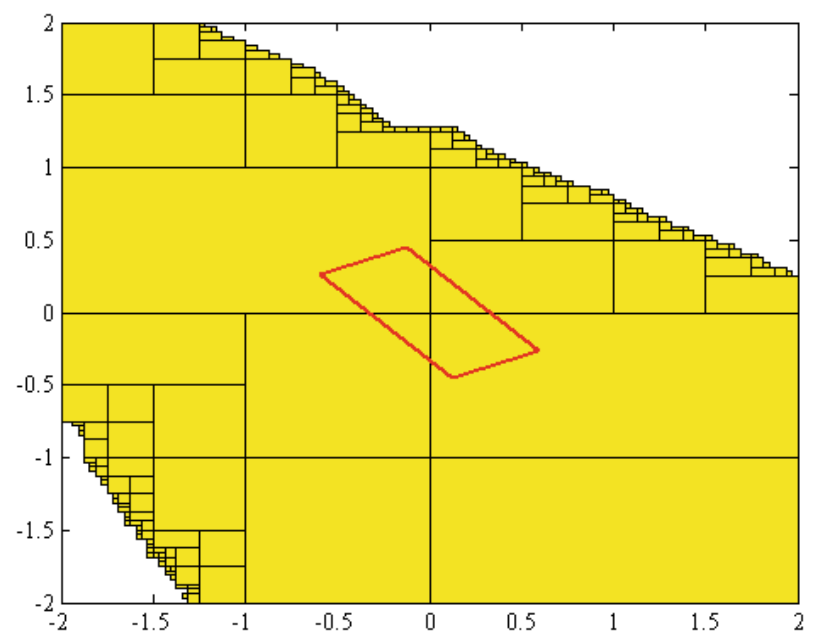

Figure 3. The inner approximation of the maximal robust controllable set with $\varepsilon=0.05$.

The dual-mode approach of model predictive control is adopted to control the system (5.1), i.e., the addressed one-step control is to drive the system state contractively along the computed robust controllable sets to the selected terminal set $\mathbb{T}$, and then the local stabilizing feedback control law is applied instead to drive the state to the origin asymptotically. Assume that the initial state is at $\mathbf{x}(0)=(1.75,-1.6)$, the resulting robust control process of the dual-mode approach of model predictive control is shown in Figure 4, where the uncertain parameter $\mu$ is a random number series between 0.85 and 0.95 during the control process and $Q=R=1$. The overall simulation time for the control process is $2248 \mathrm{~s}$, which includes the time of solving the constrained minimax optimization problem (2.4) fourteen times for obtaining one-step control inputs sequentially. It can be seen that the addressed one-step control with a sequence of robust controllable sets is guaranteed to be feasible and stable, i.e., the system can be driven contractively along the computed robust controllable sets to the selected robust control invariant set despite of an uncertain parameter during the control process. 


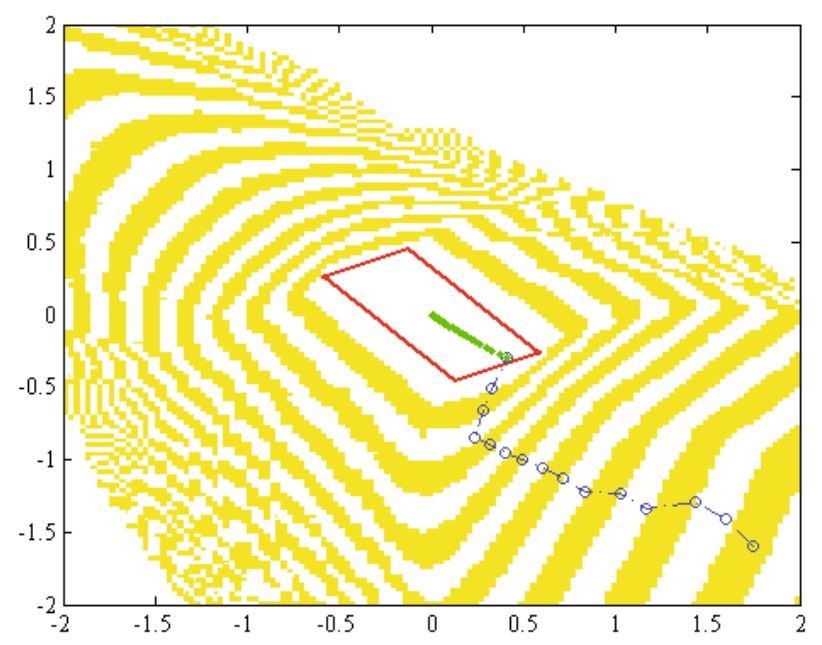

FiguRE 4. The robust control process with $\mathbf{x}(0)=(1.75,-1.6)$.

\section{Conclusions}

This paper has discussed the application of modal interval analysis to compute robust controllable sets and one-step control inputs for constrained nonlinear uncertain discrete-time systems. The solver of 1-dimensional quantified set inversion via modal interval analysis is generalized to 2-dimensional cases for computing robust controllable sets with a clear semantic interpretation. An interval-based solver of constrained minimax optimization has also been proposed to compute control inputs for the addressed one-step control approach in a reliable way. However, the burden of offline computations for robust controllable sets grows exponentially with the total dimension of the state space and the control space and extra efforts are required to judge the inclusion of the measured state to a union of interval vectors. More measures such as using polytopes to approximate the computed robust controllable sets and exploring the structure of specific nonlinear discrete-time systems are to be studied further to improve the efficiency.

Acknowledgements. This work was partially funded by the European Union and the Spanish government through the coordinated research projects DPI2004-07167-C02-02 and DPI2005-08668-C03-02 and by the government of Catalonia through SGR00296. The first author is grateful for the Ph.D. scholarship of FI-IQUC granted by the government of Catalonia, Spain. The uncommercial MATLAB Toolbox of INTerval LABoratory by Dr. Siegfried M. Rump was used in the simulation and the authors are also grateful for his implicit contribution. Additional gratitude is also owed to Dr. J.M. Bravo, Prof. M.A. Sainz and Dr. R. Calm for the discussions on the paper. The authors are also very thankful to the anonymous reviewers for their pertinent comments and valuable suggestions, which are significant for correcting and improving the paper further.

\section{REFERENCES}

[1] F. Blanchini, Set invariance in control. Automatica 35 (1999) 1747-1767.

[2] J.M. Bravo, D. Limon, T. Alamo and E.F. Camacho, On the computation of invariant sets for constrained nonlinear systems: An interval arithmetic approach. Automatica 41 (2005) 1583-1589.

[3] M. Cannon, V. Deshmukh and B. Kouvaritakis, Nonlinear model predictive control with polytopic invariant sets. Automatica 39 (2003) 1487-1494.

[4] H. Chen and F. Allgower, A quasi-infinite horizon nonlinear model predictive control scheme with guaranteed stability. Automatica 34 (1998) 1205-1217.

[5] E. Gardenes, M.A. Sainz, L. Jorba, R. Calm, R. Estela, H. Mielgo and A. Trepat, Modal intervals. Reliab. Comput. 7 (2001) $77-111$. 
[6] E. Hansen, Global Optimization Using Interval Analysis. Marcel Dekker, New York (1992).

[7] P. Herrero, M.A. Sainz, J. Vehí and L. Jaulin, Quantified set inversion algorithm with applications to control. Reliab. Comput. 11 (2005) 369-382.

[8] L. Jaulin, M. Kieffer, O. Didrit and E. Walter, Applied Interval Analysis. Springer, London (2001).

[9] E. Kaucher, Interval analysis in the extended interval space IR, Comput. Suppl. 2. Springer, Heidelberg (1980) 33-49.

[10] E.C. Kerrigan, Robust Constraint Satisfaction: Invariant Sets and Predictive Control. Ph.D. thesis, University of Cambridge, USA (2000).

[11] J. Klamaka, Controllability of nonlinear discrete systems. Internat. J. Appl. Math. Comput. Sci. 12 (2002) 173-180.

[12] W. Kühn, Rigorously computed orbits of dynamical systems without the wrapping effect. Computing 61 (1998) 47-67.

[13] D. Limon, T. Alamo and E.F. Camacho, Robust MPC control based on a contractive sequence of sets, in Proc. 42nd IEEE Conf. Dec. Control (2003) 3706-3711.

[14] D.Q. Mayne and W.R. Schroeder, Robust time-optimal control of constrained linear systems. Automatica 33 (1997) $2103-2118$.

[15] R. Moore, Interval Analysis. Prentice Hall, Englewood Cliffs, NJ (1966).

[16] S.V. Rakovic, E.C. Kerrigan and D.Q. Mayne, Reachability computations for constrained discrete-time systems with stateand input-dependent disturbances, in Proc. 42nd IEEE Conf. Dec. Control (2003) 3905-3910.

[17] S.P. Shary, A new technique in systems analysis under interval uncertainty and ambiguity. Reliab. Comput. 8 (2002) $321-418$.

[18] A.N. Sirotin and A.M. Formal'skii, Reachability and controllability of discrete-time systems under control actions bounded in magnitude and norm. Autom. Remote Control 64 (2003) 1844-1857. 